\title{
Effect of Counterions on DNA Charge Transport: A Theoretical Study
}

\author{
Yiren Wang $^{1}$, Hashem Mohammad ${ }^{1}$, M. P. Anantram ${ }^{1}$ \\ ${ }^{1}$ Department of Electrical and Computer Engineering, University of Washington, \\ Seattle, WA, USA. \\ ethanwyr@uw.edu ; hashemm@uw.edu; anantmp@uw.edu
}

\section{Extended Abstract}

DNA has been attributed as one of the leading materials in molecular nanoelectronics due to its self-assembly property and long-range charge transport. It exists in a solvent environment, surrounded by water and salt molecules. For a given DNA strand with a fixed sequence, the solvent environment becomes the most vital factor in determining its conductance. From an experimental viewpoint, researchers have measured DNA conductivity under hydrated and dehydrated conditions. However, experiments cannot easily separate the solvent contribution to conductance as some residual solvent molecules remain attached to the DNA even in the dehydrated case [1-2]. On the other hand, modeling provides the advantage of controlling the solvent environment to study its role on charge transport. Previous work has found small hydration to yield the same effects on electronic properties of the DNA as the fully hydrated one [3]. In contrast to the effect of hydration, salt counterions are not well studied, therefore raising the question about the conductance experiments: What are the implications of having/missing counterions in a hydrated or dehydrated DNA?

To precisely simulate the surrounding environment of DNA, the presence of counterions cannot be ignored. DNA molecules naturally have negative charges and hydrophilicity due to the phosphate ions in the sugar-phosphate backbone. Besides providing connections between DNA base-pairs, the backbone is also the structural support for DNA double helix shape. Meanwhile, Sodium-ion ( $\mathrm{Na}+)$, one of the most commonly used counterions, balances the negative charges, and keeps DNA molecules inside a neutral environment. Therefore, many researchers considered counterions as environmental effects along with solvent and DNA conformation, which influence DNA charge transport simultaneously. However, there are not many studies that treated counterions as an individual factor that could directly modify the DNA charge transport behavior. In this modeling study, we focus on investigating the role of $\mathrm{Na}+$ counterions within the solvent environment on charge transport through a 9 base-pair long double stranded DNA. In employing the prominent polarizable continuum model, we can change the environment from water to vacuum through changing the dielectric constant value.

Our results show that $\mathrm{Na}+$ counterions can have a significant impact on the charge transport properties of the DNA strand depending on the dielectric constant of the environment. In vacuum (low dielectric constant), the addition of Na+ lowers the bandgap from $2.57 \mathrm{eV}$ to $0.77 \mathrm{eV}$. However, the bandgap in the water case (high dielectric constant) remains almost constant at $4 \mathrm{eV}$ regardless. The reason behind this is that $\mathrm{Na}+$ adds unoccupied energy levels in the bandgap of the DNA in vacuum, while for the water case, they are added deep in the LUMO region. This can be attributed to the high dielectric constant of water, which reduces the interaction between the DNA and Na+ due to its charge screening effect. In addition, we find that the high dielectric constant increases the electronic coupling between the molecular orbitals of the DNA and yields smaller energy separation between them. Therefore, the transmission is found to be at least 2 orders of magnitude higher at HOMO and LUMO regions of the DNA in the water case. In summary, our results establish the importance of including counterions to the analysis of conductance experiments done in vacuum. As they add energy levels within the BG of the DNA in vacuum, this can further be relevant to utilizing DNA in nanoelectronics applications.

\section{References}

[1] B. Xu, P. Zhang, X. Li, and N. Tao, "Direct conductance measurement of single DNA molecules in aqueous solution," Nano Lett., vol. 4, no. 6, pp. 1105-1108, 2004, doi: 10.1021/n10494295.

[2] D. Dulić, S. Tuukkanen, C. L. Chung, A. Isambert, P. Lavie, and A. Filoramo, "Direct conductance measurements of short single DNA molecules in dry conditions," Nanotechnology, vol. 20, no. 11, 2009, doi: 10.1088/09574484/20/11/115502.

[3] M. Wolter, M. Elstner, and T. Kubař, "Charge transport in desolvated DNA," J. Chem. Phys., vol. 139, no. 12, 2013, doi: $10.1063 / 1.4821594$. 